\title{
Three-dimensional reconstruction of cone beam computed tomography images using oscar-analysis on 36 projections
}

\author{
Ramlee $\mathrm{MH}^{1 *}$, Derus $\mathrm{A}^{2}$ and Supriyanto $\mathrm{E}^{3}$ \\ ${ }^{1}$ Medical Devices and Technology Group (MEDITEG), Faculty of Biosciences and Medical Engineering, Universiti Teknologi Malaysia, Malaysia \\ ${ }^{1}$ Sports and Innovation Technology Centre (SITC), Universiti Teknologi Malaysia, Malaysia \\ ${ }^{2}$ Department of Industrial Chemistry, Faculty of Industrial Sciences and Technology, Universiti Malaysia Pahang, Lebuhraya Tun Razak, 26300 Kuantan, Pahang, Malaysia \\ ${ }^{3}$ IJN-UTM Cardio Centre, Universiti Teknologi Malaysia, Malaysia
}

\begin{abstract}
The use of Cone-Beam Computed Tomography (CBCT) scanner has become powerful tools for medical imaging techniques. This will allow medical surgeons and radiologist assistants to diagnose patients before any treatment can be taken place. However, the CBCT concepts require high demand for computer resources to reconstruct three-dimensional (3D) model from two-dimensional (2D) images. Based on this problem, Open Source Cone-Beam Reconstructor (OSCaR) was used to train medical and biomedical engineering students in understanding the concepts of computed tomography scanner. This software requires only a small capacity of computer resources, thus allowing students to practise using their own computer. With a small number of projections, the authors evaluated the performance of OSCaR to reconstruct 36 numbers of $2 \mathrm{D}$ x-ray images. By using the cone-beam $\mathrm{x}$-ray tube, 36 images of lemon and chicken bone were captured and saved into Digital Imaging and Communication in Medicine (DICOM) files. The DICOM files were then imported to the OSCaR software for the reconstruction process. Based on the results, this study successfully reconstructed $3 \mathrm{D}$ images of lemon and chicken bone. In conclusion, higher number of projections would produce better results in terms of accuracy and high resolution. However, the use of 36 numbers of $2 \mathrm{D}$ images is adequate for students to understand the concepts of computed tomography scanner.
\end{abstract}

\section{Introduction}

The basic concepts of Cone-Beam Computed Tomography (CBCT) scanner is specifically allocated in capturing images of the maxillofacial, from $2 \mathrm{D}$ images to a $3 \mathrm{D}$ model approach based on the concepts of data acquisition and image reconstruction [1]. The conebeam reconstruction can be referred as an image reconstruction from $2 \mathrm{D}$ projections data into $3 \mathrm{D}$ volume where the basic fundamental of capture through $2 \mathrm{D} \mathrm{x}$-ray area detector and cone-beam geometry. As the CBCT is able to achieve higher longitudinal resolution and relatively higher volume scan speed, many researchers have been working on it for the last two decades [2]. The first and most popular approximate reconstruction scheme for cone-beam projections acquired along a circular trajectory is the algorithm from Feldkamp and colleagues [3]. The algorithm is namely as the Feldkamp, Davis, and Kress (FDK) method which is a derivative-free method [4]. FDK conebeam reconstruction is an approximation of the exact reconstruction problem developed for technical purposes [4].

The CBCT machines possess two major concepts of scanning [2]. Firstly, the CBCT uses a low-energy fixed anode tube. This concept is similar to the dental $\mathrm{x}$-ray modalities. Second, the CBCT modalities rotate around the subject of patient, which the fundamental is capturing the data using a cone-shaped beam. All the CBCT machines that commercialized in the market often to use the same technology, but some of them possess only small differences. As far as authors concern, the major difference is the detector used in the CBCT machine[5-8]. The detector can be either an amorphous silicon flatpanel or a combination of an image intensifier and a Charge-Coupled Device (CCD) camera. Even though the detector used is different, nevertheless, both technologies have been proven to be accurate and reliable and to provide sufficient resolution for the needs of dental medicine [9]. Despite the recent development of Flat Panel Detectors (FPDs) has made cone beam CT feasible for practical use in a clinical setting, preliminary studies reported that there was limited usefulness of cone-beam CT, where it was useful only for evaluations of high contrast structures, such as high-contrast agents and bony anatomy [10].

Even though the CBCT is a small machine, however, it can expose the patients and user to approximately $20 \%$ higher radiation than a helical CT, which is equivalent to the exposure from a full-mouth periapical series [5-8]. Therefore, to allow medical and biomedical engineering students to understand the fundamental concepts of the CBCT machine, Open Source Cone-Beam Reconstructor (OSCaR) software was used in order to prevent hazards during the training purposes. The objectives of this study are; (1) to acquire $2 \mathrm{D}$ x-ray images of lemon and chicken bone using cone-beam detector and (2) to reconstruct $3 \mathrm{D}$ model of the specimens from 36 numbers of $2 \mathrm{D}$ images using OSCaR software.

Correspondence to: Muhammad Hanif Ramlee, Department of Clinical Sciences, Faculty of Biosciences and Medical Engineering, Universiti Teknologi Malaysia, Malaysia; Tel: +607-5558480; Fax.: +607-5558515; E-mail: muhammad.hanif.ramlee@biomedical.utm.my

Key words: cone beam, computed tomography, OSCaR, reconstruction, projections, DICOM

Received: December 10, 2016; Accepted: January 05, 2017; Published: January 09,2017 


\section{Material and method}

\section{Graphical user interface of OSCaR}

In the OSCaR graphical user interface, there are consists of three main stages. The first stage is the pre-processing which the CBCT data is parsed from a broad, general base of standard data-file formats such as DICOM and binary file. In addition, the geometric corrections, pixel aperture, sampling, air normalization, and other device-dependent parameters associated with the projection data are applied in this stage. For the second stage is the reconstruction. In this stage, the OSCaR permits the specification of the reconstruction filters, voxel size and Field-Of-View (FOV). The well-known FDK filtered back-projection algorithm is used to the actual voxel-driven reconstruction. The last stage is the export. For this stage, the reconstructed images and the maximum or minimum of the reconstructed volume can be saved in $a^{*}$.mat file [13].

\section{Resolution and geometry parameter}

In order to acquire the 2D images data of lemon and chicken bone, the below parameters that describe the geometry and resolution of cone-beam apparatus were recorded before the scanning process can be conducted:

$d u$ - Pixel length $(\mathrm{cm})$ in $u$ direction (perpendicular to the axis).

$v$ - Pixel length $(\mathrm{cm})$ in $v$ direction (parallel to the axis).

$S A D$ - Source-Axis-Distance $(\mathrm{cm})$.

$S D D$ - Source-Detector-Distance $(\mathrm{cm})$.

This parameter is required for setting purposes at the Graphical User Interface (GUI) OSCaRPreprocessing before can be proceed in the reconstruction process. Table 1 illustrated the resolution and geometry parameters before the scanning process are taken place.

\section{Projection-dependent parameter}

To be noted, certain parameters can vary from projection to projection. The projection has been specified and saved to csv (comma separated values) file for the use of GUI OSCaRPreprocess. In this stage, a csv file was prepared and later will be exported to the OSCaR program. In this study, the .csv files were consisted of Nproj rows, each with 6 columns. The 6 columns of the kth row of the csv file consist of information pertinent to the kth projection $(\mathrm{k}=1$ : Nproj) [13]. These columns are as follows:

filename $\left|\theta_{\mathrm{G}}\right| u_{\text {off }}\left|v_{\text {off }}\right| I_{o} \mid w$

Filename : string naming file in which $k$ th projection is stored

$\theta_{\mathrm{G}} \quad$ : gantry angle of $k$ th projection (degrees)

$u_{\text {off }}:$ offset of centre of detector perpendicular to axis $(\mathrm{cm})$

$v_{\text {off }}:$ offset of centre of detector parallel to axis $(\mathrm{cm})$

$I_{o} \quad$ : air normalisation (same units as values in projections)

w : weight (dimensionless)

Table 1. Resolution and geometry parameters.

\begin{tabular}{|c|c|}
\hline Parameters & Value $(\mathrm{cm})$ \\
\hline$d u$ & 0.1552 \\
\hline$d v$ & 0.1552 \\
\hline$S A D$ & 100 \\
\hline$S D D$ & 5 \\
\hline
\end{tabular}

\section{Types of phantom and degree of projections}

This study used two types of phantom; lemon and chicken bone. The use of small phantom is preferable as the MATLAB program could not support a big size of images. In this study, the images consisted of 36 projections which the intervals were 10 degrees for each projection (e.g. $10^{\circ}, 20^{\circ}, 30^{\circ}$ ).

\section{Characteristic of detector and generator power}

For acquiring the $2 \mathrm{D}$ images of lemon and chicken bone, the PaxScan 4336R detector was used in this study. This detector is the first ruggedized portable X-ray flat panel detector designed for mobile digital radiographic X-ray systems to fit existing 14"x17" standard trays. Based upon the new Gigabit Ethernet interface, images are displayed on a user-supplied workstation. For the best quality of 2D images, the generator of $\mathrm{x}$-ray tube was set to $50 \mathrm{kV}$ and $12 \mathrm{~mA}$ for lemon, and 40 $\mathrm{kV}$ and $16 \mathrm{~mA}$ was for chicken bone.

\section{Project flow}

Reconstructions process using OSCaR requires several steps before obtaining the results. Figure 1 shows an overview of reconstruction steps.

\section{Results and discussion}

\section{Images from raw data}

Collecting data from X-Ray CT scanner consists of various types of format. In order to reduce loss of image information, therefore all the images were saved into .raw file after the data acquisition. Figure 2

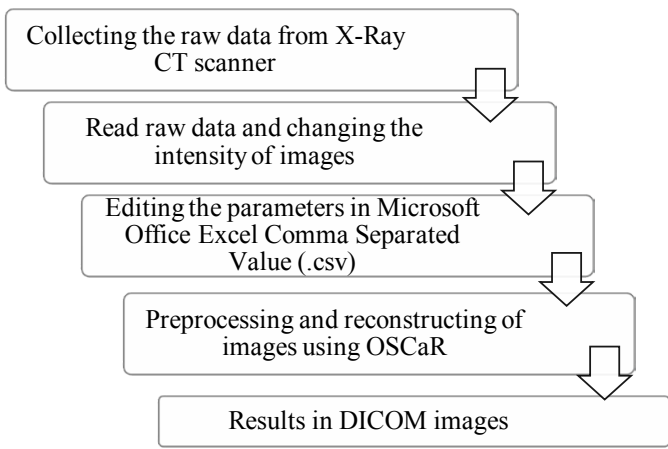

Figure 1. Overview of work flow.

a)

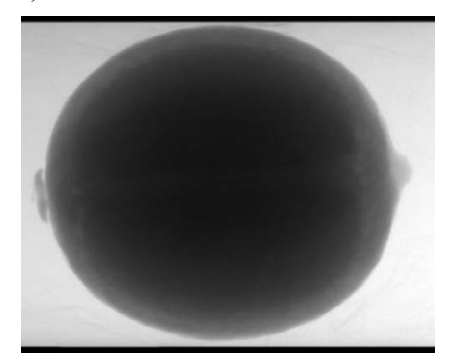

b)

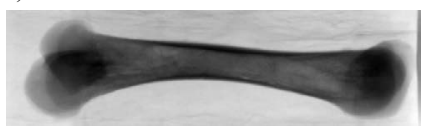

Figure 2. The 2D images of (a) lemon and b) chicken bone. 
shows $2 \mathrm{D}$ images of lemon and chicken bone. This image was at zero degrees of projections angle.

\section{Reconstruction images}

Figure 3 shows the reconstruction results for the phantom of lemon in the axial view. After the reconstruction process done using OSCaR, there were 60 slices of DICOM images

The internal region indicates the size of lemon according to their number of slices and the other regions was a noise caused from the reconstruction process. To be noted, the slice number 25 and 30 indicated a full size of the lemon in axial view. Figure 4 shows the results of the chicken bone after the reconstruction process. There were 50 numbers of slices in DICOM images (axial view).

Based on the results, the reconstructed images for the chicken bone is clear at the slice 19 until 28. As compared with the reconstructed images of lemon, the resolution and accuracy of image reconstruction for the chicken bone are much lower. This can be seen that the rest of the region shows many noises [5]. This situation cannot be avoided since the number of $2 \mathrm{D} \mathrm{X}$-ray images was only 36 . Therefore, for the best results in terms of accuracy and high resolution, the number of images should be increased [6].

\section{Reconstruction in 3D slicer}

The 3D Slicer was used to represent all the DICOM images into 3D view after reconstructed. Figure 5(a) and 5(b) indicated the 3D view of image reconstruction in axial, saggital and coronal view for lemon and chicken bone, respectively. However, the results were not appearing in a volume of the materials used. From the image in the Figure 5(a) and 5(b), it can be seen that the soft tissues inside the lemon and hard tissues inside the chicken bone.

\section{Conclusion}

Based on the results, reconstruction of cone-beam computed tomography (CBCT) images using OSCaR for 36 numbers of a)
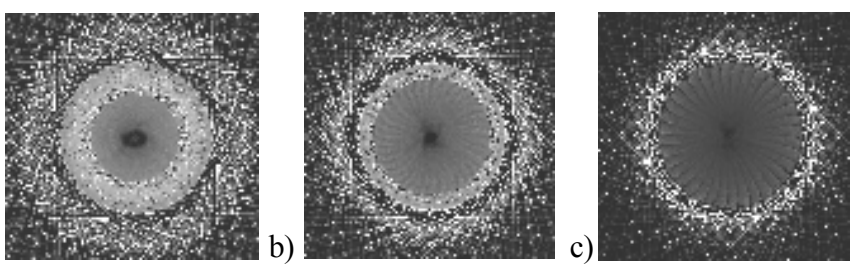

d)
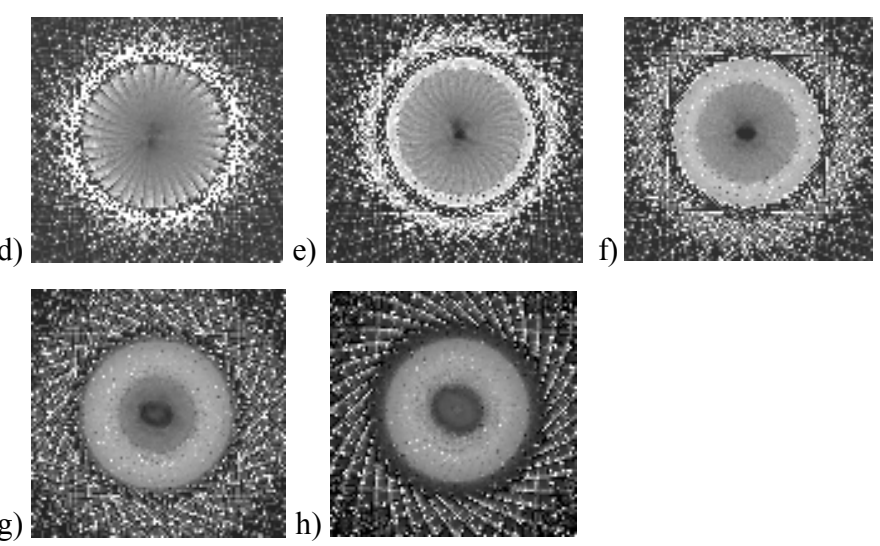

Figure 3. The reconstructed images of lemon in the axial view for (a) Slice 15, (b) Slice 20, (c) Slice 25, (d) Slice 30, (e) Slice 35, (f) Slice 40, (g) Slice 45 and (h) Slice 50.

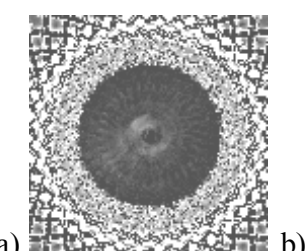

a)
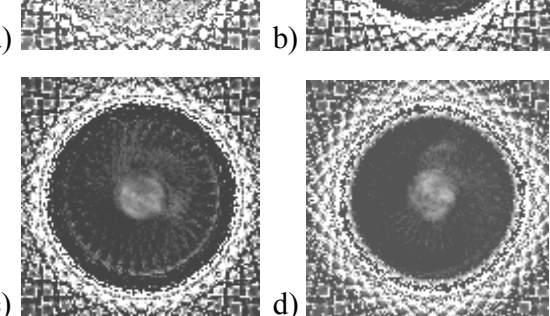

c)

e)
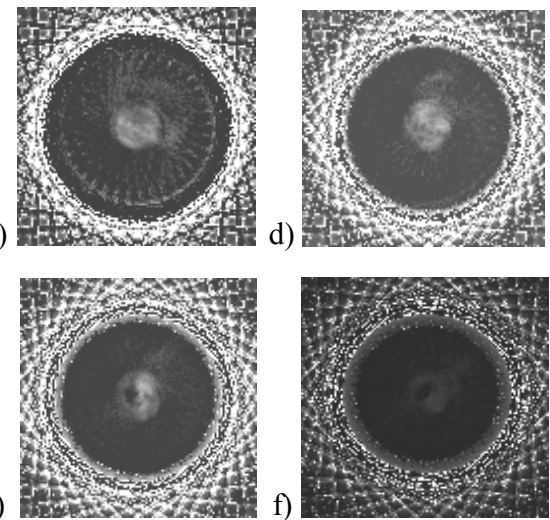

g)
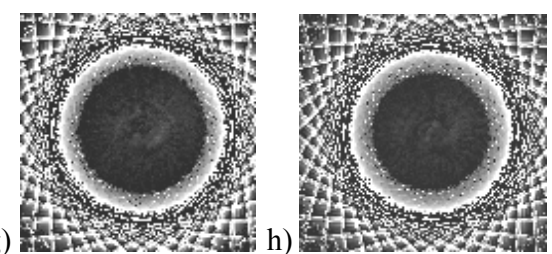

Figure 4. The reconstructed images of chicken bone in the axial view for the (a) Slice 14 (b) Slice 19, (c) Slice 25, (d) Slice 28, (e) Slice 30, (f) Slice 32, (g) Slice 34, (h) Slice 35.
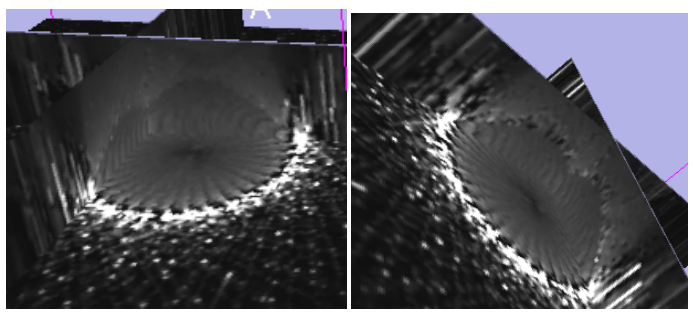

(a)

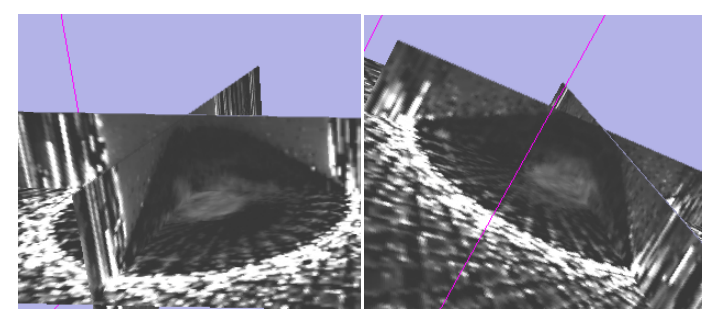

(b)

Figure 5. Reconstruction images in 3D Slicer (combined axial, saggital and coronal view) a) lemon at slice 33, b) chicken bone at slice 28 .

projections is successful. However, with a limited number of computer resources and projections, the 3D image of phantoms seen not in a clear view. Therefore, it is recommended that further research can be conducted in applying a higher number of projections for better image reconstruction. 


\section{Acknowledgement}

The authors would like to express thankfulness to all who have contributed through their wide range of support to the success of this work. Special thanks to Prof. Dr. Ing Habil Jens Haueisen and Dr. Dunja Jannek from Ilmenau University of Technology, Germany for their supporting, comments and which has enriched authors with their dedicated care and constant willingness to discuss with a wide range of information for thought and assisted in preparing this study. We also like to thank the Ilmenau University of Technology, Germany and Universiti Teknologi Malaysia for the financial support to the students who conducted this project.

\section{References}

1. Scarfe WC, Farman AG (2008) What is Cone-Beam CT and How Does it Work? Dent Clin North Am 52:707-730. [Crossref]

2. Zeng K (2004) Review of Recent Developements in Cone-Beam CT Reconstruction Algorithm for Long-object Problem. Image Anal Stereol 23: 83-87.

3. Feldkamp LA, Davis LC, Kress JW (1984) Practical cone-beam algorithm. J Opt Soc Am A1: 612-619.

4. Buzug TM (2008) Computed Tomography: From Photon Statistics to Modern ConeBeam CT Springer 371.
5. Mah J, Hatcher D (2004) Three-dimensional craniofacial imaging. Am J Orthod Dentofac Orthop 126: 308. [Crossref]

6. Kunihiko S, Kazuo Y, Kan U (2002) Development of dentomaxillofacial cone beam X-ray CT system model CB MercuRay. Medix 37: 40.

7. Palomo JM, Kau CH, Bahl Palomo L, et al. (2006) Three-dimensional cone beam computerized tomography in dentistry. Dent Today 25: 130. [Crossref]

8. Sukovic P (2003) Cone beam computed tomography in craniofacial imaging. Orthod Craniofac Res 6:31. [crossref]

9. Quereshy FA, Savell TA, Palomo JM (2008) Applications of Cone Beam Computed Tomography in the Practice of Oral and Maxillofacial Surgery. J Oral Maxillofac Surg 66: 791-796.

10. S Kakeda (2007) A Cone-Beam Volume CT Using a 3D Angiography System with a Flat Panel Detector of Direct Conversion Type: Usefulness for Superselective Intraarterial Chemotherapy for Head and Neck Tumors. AJNR Am J Neuroradiol 28: 17831788. [Crossref]

11. Dula K, Mini R, van der Stelt PF, Lambrecht JT, Schneeberger P, et al. (1996) Hypothetical mortality risk associated with spiral computed tomography of the maxilla and mandible. Eur J Oral Sci 104: 503-510. [Crossref]

12. Brooks SL (2005) Effective dose of two cone-beam CT scanners:, "i-CAT and NewTom 3G", Quarterly Publication of the American Association of Dental Maxillofacial Radiographic Technicians, Winter 2005.

13. Nargol Rezvani (2008) Open Source Cone-beam Reconstructor.

Copyright: $₫ 2016$ Ramlee MH. This is an open-access article distributed under the terms of the Creative Commons Attribution License, which permits unrestricted use, distribution, and reproduction in any medium, provided the original author and source are credited. 